\title{
The impact of genetic variants in inflammatory-related genes on prostate cancer risk among men of African Descent: a case control study
}

\author{
Dominique Z Jones ${ }^{1}$, Camille Ragin², Nayla C Kidd ${ }^{1}$, Rafael E Flores-Obando ${ }^{3}$, Maria Jackson ${ }^{4}$, \\ Norma McFarlane-Anderson ${ }^{5}$, Marshall Tulloch-Reid ${ }^{6}$, Kevin S Kimbro ${ }^{7}$ and LaCreis R Kidd ${ }^{{ }^{*}}$
}

\begin{abstract}
Purpose: Although case-control studies have evaluated the role of variant inflammatory-related loci in prostate cancer, their impact is virtually unknown among men of African descent. To address this, we evaluated the impact of inflammatory cytokine single nucleotide polymorphisms (SNPs) on prostate cancer risk for men of African descent.

Methods: Forty-four SNPs in inflammatory cytokine-associated genes were evaluated among 814 African-American and Jamaican men (279 prostate cancer cases and 535 controls) using Illumina's Golden gate genotyping system. Individual SNP effects were evaluated using logistic regression analysis.

Results: Four SNPs were modestly associated with prostate cancer after adjusting for age. In the total population, inheritance of the ILIR2 rs11886877 AA, IL8RB rs11574752 AA, TNF rs1800629 GA + AA, and TNF rs673 GA genotypes modestly increased prostate cancer risk by 1.45 to 11.7 -fold relative to the referent genotype. Among U.S. men, age-adjusted dominant, recessive and additive genetic models for the ILIR2 rs 11886877 locus were linked to an increase in prostate cancer susceptibility. However, these main effects did not persist after adjusting for multiple hypothesis testing.
\end{abstract}

Conclusion: Our preliminary data does not strongly support the hypothesis that inflammatory-related sequence variants influence prostate cancer risk among men of African descent. However, further evaluation is needed to assess whether other variant inflammatory-related genes may contribute to prostate cancer risk and disease progression in larger and ethnically diverse multi-center studies.

Keywords: Prostate cancer, Inflammatory-related sequence variants, Single nucleotide polymorphisms

\section{Introduction}

Chronic inflammation is thought to predispose an individual to cancer development [1]. This relationship is supported by a number of studies involving inflammatory bowel disease, colon cancer, hepatitis, liver cancer, pancreatitis, and pancreatic cancer [2-6]. Through several lines of evidence from epidemiological, histopathological, animal, genetic and molecular pathological studies, chronic inflammation

\footnotetext{
* Correspondence: Irkidd01@louisville.edu

'Department of Pharmacology \& Toxicology, University of Louisville, Louisville, KY, USA

Full list of author information is available at the end of the article
}

is also thought to play a major role in prostate cancer development [2,3]. For example, prostatic infections have been implicated in prostate cancer either through direct or indirect promotion of the inflammatory process [1-3]. In addition, the use of non-steroidal anti-inflammatory drugs (NSAIDS) and other anti-inflammatory agents have been shown to reduce prostate cancer risk [4].

The production of cytokines can be influenced by single nucleotide polymorphisms (SNPs) detected within pro- and anti-inflammatory genes. Genetic variations in cytokine related genes may lead to alterations in the spectrum of cytokines expressed in an inflammatory environment or

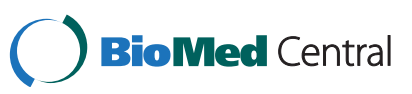


level of antitumor response [5]. Epidemiological studies have reported on the relationship between prostate cancer susceptibility and genes involved in the cytokine-cytokine receptor signaling pathway, such as interleukins and their receptors, ribonuclease $\mathrm{L}$ (RNASEL) and tumor necrosis factor (TNF) [6-13]. While men of African Descent suffer disproportionately from this disease $[2,3,14,15]$, there is limited information about the positive link between variant cytokine genes and prostate cancer development in this population [16,17]. Therefore, additional studies are needed to investigate the role of inflammatory-related SNPs in the development of prostate cancer among individuals of African Descent.

The current study evaluated the impact of 44 inflammatory-related sequence variants in relation to prostate cancer risk among men of African Descent from the U.S. and Jamaica. Findings from our study will help to fill in the gaps in information pertinent to prostate cancer among men of African Descent.

\section{Materials and methods Study population}

Our study population, 279 cases and 535 controls, was comprised of two independent case-control study sets. These studies include the Prostate Cancer Clinical Outcome Study ( $\left.\mathrm{PC}^{2} \mathrm{OS}\right)$ at the University of Louisville and the Prostate Cancer Study in Jamaica at the University of the West Indies, Mona Campus. For both study sets, all incident prostate cancer cases were histologically confirmed and the controls were assigned based on normal PSA levels, and normal DREs/biopsies. Descriptions of each contributing study have been previously described $[18,19]$. Briefly, the $\mathrm{PC}^{2} \mathrm{OS}$ study included 170 incident prostate cancer cases and 433 controls recruited between 2001-2005 through the Howard University Hospital (HUH) Division of Urology or related prostate cancer screening programs. Enrolled participants were men of African descent from the Washington, D.C. and Columbia, S.C. areas. The racial subgroups included self-reported African Americans, East African Americans, West African Americans, and Caribbean Americans. The Prostate Cancer Study in Jamaica included consecutively enrolled 109 incident prostate cancer cases and 102 controls recruited from 2005-2007 through the Urology clinic at the University Hospital of the West Indies in Kingston Jamaica.

\section{Criteria for inflammatory gene and SNP selection}

Inflammatory-associated genes and SNPs were selected using one or more of the following criteria: (1) empirical evidence that supports a relationship between the SNP/ gene and cancer or inflammatory/immune response related diseases; (2) commonly studied loci; (3) marked disparities in genotype frequency comparing men of African Descent to their Caucasian counterparts (i.e., $\pm 10 \%$ change); (4) evidence demonstrating a link with alterations in mRNA expression/stability or protein expression/structure or function using in silico tools such as SNPinfo (http:// snpinfo.niehs.nih.gov/snpinfo/snpfunc.htm) or published reports; and (5) a minor allele frequency $\geq 5 \%$ reported in the National Center for Biotechnology Information (NCBI) Entrez SNP, (http://www.ncbi.nlm.nih.gov/snp). According to NCBI, the selected SNPs had an average minor allele frequency of $22 \%$. However, the IL1RN rs315951 SNP had an allele frequency of $2.1 \%$. This rare non-synonymous sequence variant was included in the analysis to explore whether a rare SNP would lead to substantial gains in effect sizes (i.e., 2-3 fold increases in risk) and contribute to the missing genetic heritability $[20,21]$.

\section{Genetic analysis of variant inflammatory-associated SNPs}

Allelic discrimination of 44 inflammatory-associated sequence variants was performed using a custom Illumina GoldenGate Genotyping assay with VeraCode Technology and BeadXpress reader, according to the manufacturer's instructions [22].

\section{Statistical analysis}

Evaluation of the relationship between variant inflammatory associated alleles and prostate cancer risk was performed using univariate and multivariate analyses. The chi-square test of heterogeneity was used to assess for significant differences in the distribution of homozygous major, heterozygous, or homozygous minor genotypes between prostate cancer cases and controls. Evaluation of the relationship between prostate cancer risk and selected polymorphic genes, expressed as odds ratios (ORs) and corresponding 95\% confidence intervals (CIs), were estimated using unconditional multivariate LR models adjusted for age. The major or common genotype was used as the reference category for each LR model. Statistical significance was assessed using a Bonferroni Correction $(\alpha=0.05 / 44$ SNPs) cut-off of 0.001 , in order to adjust for multiple comparisons. All statistical analyses were performed using SAS 9.3 (SAS Institute Inc., Cary, NC) and SNP Variation Software 7.0 (Golden Helix, Bozeman, MT).

\section{Statistical power}

Based on our sample size for the total population, U.S. and Jamaican men, we had $>80 \%$ power to detect SNPs with odds ratios (ORs) of $\geq 1.4, \geq 1.6, \geq 1.8$, respectively, for a co-dominant genetic model with 1 degree of freedom (df), a minor allele frequency of at least $22 \%$ and disease prevalence of $0.74 \%$. Analyses were performed using Power for Genetic Association Version 2 Software [23]. 


\section{Results}

Prevalence of inflammatory-associated sequence variants among men of African Descent

Inheritance of variant inflammatory-related loci was fairly common among African-American men in the current study. Specifically, the minor allele frequencies of the 44 sequence variants ranged from approximately $2.6 \%$ to $48 \%$, as depicted in Table 1 . Notably, the observed genotype frequency distribution among controls did not significantly deviate from expected counts according to the Hardy Weinberg equilibrium. With the exception of four loci (IL1RN rs4251961, IL10RB rs999788, IL10RB rs283416, and ILR1 rs3917225), the observed genotype frequencies in the current study corroborated with values for individuals of African-American/African ancestry reported in the NCBI's SNP entrez $(\mathrm{P}=0.063-1.000)$, as shown in Table 1.

\section{Relationship between inflammatory sequence variants and prostate cancer risk}

Seven out of 44 sequence variants detected in inflammatory-related genes were modestly associated with prostate cancer risk among 814 men of African Descent (279 cases and 535 controls), as summarized in Table 2. For age-adjusted risk models, elevations in prostate cancer susceptibility were observed among carriers of IL1R2 rs1188687 7AA $(\mathrm{OR}=1.92 ; 95 \% \mathrm{CI}=1.11,3.32), I L 8 R B$ $\mathrm{rs} 11574752 \mathrm{GA}+\mathrm{AA}(\mathrm{OR}=38.40 ; 95 \% \mathrm{CI}=3.86,382.8)$, TNF rs1800629 GA + AA (OR = 1.53; 95\%CI = 1.06, 2.20), and $T N F$ rs673 GA $(\mathrm{OR}=1.50 ; 95 \% \mathrm{CI}=1.04,2.16)$ genotypes with risk estimates ranging from 1.50-38.4. The IL1R2 rs11886877 marker was the only genetic susceptibility factor significant under the additive genetic model (P-trend $=0.010)$, indicative of a significant dose-response effect in relation to the number of inherited minor alleles. The aforementioned markers were not classified as important prostate cancer risk indicators after adjusting for multiple comparisons bias using the Bonferroni correction, with a significance cut-off of $\leq 0.001$.

Upon stratification by sub-population, modestly significant prostate cancer biomarkers varied by racial/ethnic group in the age adjusted risk models. Possession of the RNASEL rs1213524 AG genotype was associated with a 2.17-fold increase in the risk of developing prostate cancer $(\mathrm{OR}=2.10 ; 95 \% \mathrm{CI}=1.04,4.24)$ among Jamaican men, as detailed in Table 3. However, this locus was not significant in the dominant, recessive or additive genetic models. Similar to the total population, inheritance of sequence variants in IL1R2, IL1ORA and TNF among U.S. men were linked with a significant increase in prostate cancer risk. Among U.S. men, two inflammatory-related sequence variants, [IL1R2 rs11886877 (GA, GA + AA, AA) and IL1ORA rs4252243 AA], were associated with a 1.82-2.49-fold increase in prostate cancer risk. Out of these 2 markers, the
IL1R2 rs11886877 locus was significant for the dominant $(\mathrm{OR}=2.75 ; 95 \% \mathrm{CI}=1.38,5.50)$, co-dominant $(\mathrm{OR}=1.82$; $95 \% \mathrm{CI}=1.14,2.88)$, recessive $(\mathrm{OR}=2.05 ; 95 \% \mathrm{CI}=1.10$, 3.80 ), and additive ( $\mathrm{P}$-trend value $=0.002$ ) genetic models. None of the aforementioned markers survived correction for multiple hypotheses testing. Moreover, the IL10RA rs4252243 SNP was only significantly related to prostate cancer risk under the recessive genetic model $(\mathrm{OR}=2.49$; $95 \% \mathrm{CI}=1.08,5.72$ ).

\section{Discussion}

Chronic inflammation has been associated with tumor development and metastasis. Inflammatory response is regulated through a complex network of cytokines, cytokine receptors and downstream targets that synergistically regulate innate/humoral immune and inflammatory processes. Recent molecular and genetic epidemiology studies have demonstrated that chronic inflammation and susceptibilities in inflammatory-associated genes are related to the development of several cancers, including lymphoma, and gastric and prostate cancer [6,16,24-26]. However, to our knowledge, there are few published reports on the impact of variant cytokine-related genes in relation to prostate cancer among men of African Descent. Therefore, the current study evaluated the individual effects of 44 inflammatory associated sequence variants on prostate cancer risk among 279 cases and 535 disease-free men of African Descent from the U.S and Jamaica. Our findings revealed a modest increase in prostate cancer risk for unadjusted and adjusted logistic regression models for IL1R2 rs11886877 among men of African Descent. The additive, dominant and recessive genetic models of this variant were significant even after adjusting for age. However, this relationship did not survive after accounting for multiple comparisons bias.

IL1R2 rs11886877 is about 2415 base pairs from the transcription start site, which suggest it may have a high likelihood of regulating IL1R2 gene expression. Currently, there are no published reports on the relationship between IL1R2 rs11886877 and prostate cancer for any population. Although there is no evidence of the impact of this sequence variant on prostate cancer risk among European and African American men, the relationship between the IL1R2 gene expression and prostate cancer has been demonstrated through published reports [27-29]. Leshem and colleagues (2011) found that the promoter region of IL1R2 possesses putative binding motifs for the TMPRSS2/ERG fusion gene, which is highly expressed in aggressive prostate cancer [27]. When the expression of IL1R2 was knocked down using small interfering RNAs, it resulted in the reduction of ZEB2 mRNA expression in hTERT/ shp53/CyclinD-CDK4 overexpressing cells exposed to TMPRSS2/ERG [25]. TMPRSS2/ERG fusion gene indirectly up-regulates ZEB2, a facilitator of the epithelial to 
Table 1 Functional consequence and prevalence of inflammatory-associated sequence variants

\begin{tabular}{|c|c|c|c|c|c|c|c|c|c|c|c|c|c|}
\hline dbSNPID & Gene & $\begin{array}{l}\text { Location } \\
\text { functional } \\
\text { consequence }\end{array}$ & $\begin{array}{l}\text { NCBI } \\
\text { nucleotide } \\
\text { change } \\
\text { (major }>^{\text {minor allele) }}{ }^{\dagger}\end{array}$ & $\begin{array}{l}\mathrm{NCBI} \\
\text { minor allele } \\
\text { frequency } \\
\text { (MAF) for } \\
\text { African- } \\
\text { Americans }\end{array}$ & $\begin{array}{l}\mathrm{NCBI} \\
\text { major/major } \\
\text { genotype } \\
\mathrm{n}(\%) \text { for } \\
\text { African- } \\
\text { Americans }\end{array}$ & $\begin{array}{l}\text { NCBI } \\
\text { major/minor } \\
\text { genotype } \\
n \text { (\%) for } \\
\text { African- } \\
\text { Americans }\end{array}$ & $\begin{array}{l}\text { NCBI } \\
\text { minor/minor } \\
\text { genotype } \\
\mathrm{n}(\%) \text { for } \\
\text { African- } \\
\text { Americans }\end{array}$ & $\begin{array}{l}\text { Current study } \\
\text { nucleotide } \\
\text { change } \\
\text { (major > } \\
\text { minor allele) }\end{array}$ & $\begin{array}{l}\text { Current } \\
\text { study MAF } \\
\mathrm{n}(\%) \text { for } \\
\text { African- } \\
\text { Americans }\end{array}$ & $\begin{array}{l}\text { Current } \\
\text { study } \\
\text { major/major } \\
\text { genotype } \\
\mathrm{n}(\%) \text { for } \\
\text { African- } \\
\text { Americans }\end{array}$ & $\begin{array}{l}\text { Current } \\
\text { study } \\
\text { major/minor } \\
\text { genotype } \\
\mathrm{n}(\%) \text { for } \\
\text { African- } \\
\text { Americans }\end{array}$ & $\begin{array}{l}\text { Current } \\
\text { study } \\
\text { minor/minor } \\
\text { genotype } \\
\mathrm{n}(\%) \text { for } \\
\text { African- } \\
\text { Americans }\end{array}$ & $\begin{array}{l}\text { Overall } X^{2} \\
\text { P-value } \\
\text { comparing } \\
\text { genotypes from } \\
\text { individuals of } \\
\text { African Descent } \\
\text { as reported in } \\
\text { NCBI versus the } \\
\text { current study }{ }^{+1}\end{array}$ \\
\hline rs1058867 & IL10RB & $\begin{array}{l}\text { UTR'3 } \\
\text { miRNA }\end{array}$ & $G>A$ & $A=37.9$ & $26(42.0)$ & $25(40.3)$ & $11(17.7)$ & $G>A$ & $A=33.9$ & 239 (44.6) & $229(42.9)$ & $67(12.5)$ & 0.514 \\
\hline rs $1071676^{\ddagger}$ & IL1B & $\begin{array}{l}\text { UTR'3 } \\
\text { miRNA }\end{array}$ & $G>C$ & $C=14.6$ & 19 (79.2) & $3(12.5)$ & $2(8.3)$ & $G>C$ & $C=16.1$ & $378(70.7)$ & $142(26.5)$ & $15(2.80)$ & 0.106 \\
\hline rs11123902 ${ }^{\ddagger}$ & IL1R2 & Intron 1 & $A>C$ & $C=31.8$ & $10(45.5)$ & $10(45.5)$ & $2(9.10)$ & $A>C$ & $C=30.7$ & $258(48.2)$ & 225 & $52(9.70)$ & 0.949 \\
\hline rs1126579 & IL8RB & $\begin{array}{l}\text { UTR'3 } \\
\text { miRNA }\end{array}$ & $C>T$ & $T=14.5$ & $46(74.2)$ & $14(22.6)$ & $2(3.20)$ & $G>A^{+}$ & $A=13.8$ & $402(75.1)$ & $118(22.1)$ & $15(2.80)$ & 0.886 \\
\hline rs1143627 & IL1B & $\begin{array}{l}\text { Near gene } 5^{\prime} \\
\text { TFBS }\end{array}$ & $C>T$ & $\mathrm{~T}=37.5$ & $12(50.0)$ & $6(25.0)$ & $6(25.0)$ & $G>A^{\dagger}$ & $A=39.6$ & $194(36.3)$ & $256(48.2)$ & $83(15.5)$ & 0.063 \\
\hline rs1143634 & IL1B & $\begin{array}{l}\text { Exon } 4 \\
\text { Splicing }\end{array}$ & $C>T$ & $\mathrm{~T}=12.9$ & $47(75.8)$ & $14(22.6)$ & $1(1.60)$ & $G>A^{\dagger}$ & $A=15.5$ & $381(71.2)$ & $142(26.5)$ & $12(2.3)$ & 0.833 \\
\hline rs11574752 & IL8RB & $\begin{array}{l}\text { UTR'3 } \\
\text { miRNA }\end{array}$ & $G>A$ & $A=10.4$ & $19(79.2)$ & $5(20.8)$ & $0(0.00)$ & $G>A$ & $A=9.40$ & 435 (81.3) & 99 (18.5) & $1(0.20)$ & 0.798 \\
\hline rs11886877 & IL1R2 & Intron 1 & - & - & - & - & - & $G>A$ & $A=35.8$ & $211(39.4)$ & 265 (49.5) & 59 (11.1) & \\
\hline rs12135247 & RNASEL & $\begin{array}{l}\text { UTR'3 } \\
\text { TFBS, miRNA }\end{array}$ & $\mathrm{T}>\mathrm{C}$ & $C=16.3$ & $33(67.3)$ & $16(32.7)$ & $0(0.00)$ & $A>G^{+}$ & $G=17.9$ & $368(68.8)$ & $142(26.5)$ & $25(4.70)$ & 0.226 \\
\hline rs12328606 & IL1R2 & $\begin{array}{l}\text { Near gene } 5^{\prime} \\
\text { TFBS }\end{array}$ & $C>T$ & $\mathrm{~T}=11.2$ & $38(77.6)$ & $11(22.4)$ & $0(0.00)$ & $G>A^{\dagger}$ & $A=13.5$ & $405(75.7)$ & $116(21.7)$ & $14(2.60)$ & 0.798 \\
\hline rs $1304037^{\ddagger}$ & IL1A & $\begin{array}{l}\text { UTR'3 } \\
\text { miRNA }\end{array}$ & $A>G$ & $G=39.6$ & $8(33.3)$ & $13(54.2)$ & $3(12.5)$ & $A>G$ & $G=41.1$ & 191 (35.7) & $248(46.4)$ & $96(17.9)$ & 0.760 \\
\hline rs $16944^{\ddagger}$ & IL1B & $\begin{array}{l}\text { Near gene } 5^{\prime} \\
\text { TFBS }\end{array}$ & $A>G$ & $G=39.0$ & $18(30.5)$ & $36(61.0)$ & $5(8.50)$ & $A>G$ & $G=45.1$ & $156(29.2)$ & 275 (51.4) & 104 (19.4) & 0.108 \\
\hline rs $17561^{\neq}$ & IL1A & $\begin{array}{l}\text { Exon } 4 \\
\text { Splicing, } \\
\text { nsSNP, } \\
\text { benign }\end{array}$ & $G>T$ & $T=15.3$ & $44(71.0)$ & $17(27.4)$ & $1(1.60)$ & $C>A^{\dagger}$ & $A=18.6$ & $358(66.9)$ & $155(29.0)$ & $22(4.10)$ & 0.698 \\
\hline rs1799964 & TNF & $\begin{array}{l}\text { Near gene } 5^{\prime} \\
\text { TFBS }\end{array}$ & $T>C$ & $C=12.9$ & $46(74.2)$ & 16 (25.8) & $0(0.00)$ & $A>G^{\dagger}$ & $G=16.5$ & 374 (69.9) & $145(27.1)$ & $16(3.00)$ & 0.492 \\
\hline rs $1800587^{\ddagger}$ & IL1A & $\begin{array}{l}\text { UTR'5 } \\
\text { TFBS, Splicing }\end{array}$ & $C>T$ & $T=39.1$ & $9(39.1)$ & $10(43.5)$ & $4(17.4)$ & $G>A^{\dagger}$ & $A=41.8$ & $183(34.2)$ & $257(48.0)$ & $95(17.8)$ & 0.885 \\
\hline rs1800629 & TNF & $\begin{array}{l}\text { Near gene } 5^{\prime} \\
\text { TFBS }\end{array}$ & $G>A$ & $A=13.7$ & $46(74.2)$ & $15(24.2)$ & $1(1.60)$ & $G>A$ & $A=16.9$ & $368(68.8)$ & 153 (28.6) & $14(2.60)$ & 0.752 \\
\hline rs $1800871^{\neq}$ & IL10 & $\begin{array}{l}\text { Near gene } 5^{\prime} \\
\text { TFBS }\end{array}$ & $C>T$ & $T=36.3$ & $28(45.2)$ & $23(37.1)$ & $11(17.7)$ & $G>A^{\dagger}$ & $A=40.7$ & $188(35.0)$ & $258(48.0)$ & 89 (17.0) & 0.219 \\
\hline
\end{tabular}


Table 1 Functional consequence and prevalence of inflammatory-associated sequence variants (Continued)

\begin{tabular}{|c|c|c|c|c|c|c|c|c|c|c|c|c|c|}
\hline rs $1800872^{\neq \neq}$ & IL10 & $\begin{array}{l}\text { Near gene } 5^{\prime} \\
\text { TFBS }\end{array}$ & $A>C$ & $\begin{array}{l}A=50.0 \\
C=50.0\end{array}$ & $5(21.7)$ & 13 (56.6) & $5(21.7)$ & $C>A^{t}$ & $A=40.7$ & $188(35.0)$ & $258(48.0)$ & 89 (17.0) & 0.874 \\
\hline rs $1800893^{\neq}$ & IL10 & $\begin{array}{l}\text { Near gene } 5^{\prime} \\
\text { TFBS }\end{array}$ & $G>A$ & $A=37.1$ & $22(35.5)$ & $34(54.8)$ & $6(9.70)$ & $G>A$ & $A=36.4$ & $216(40.4)$ & $248(46.4)$ & $71(13.2)$ & 0.419 \\
\hline rs $1800896^{\ddagger}$ & IL10 & $\begin{array}{l}\text { Near gene } 5^{\prime} \\
\text { TFBS }\end{array}$ & $A>G$ & $G=40.5$ & $7(33.3)$ & $11(52.4)$ & $3(14.3)$ & $A>G$ & $G=33.3$ & $243(45.4)$ & $228(42.6)$ & 64 (12.0) & 0.491 \\
\hline rs2192752 ${ }^{\ddagger}$ & IL1R1 & $\begin{array}{l}\text { Near gene } 5^{\prime} \\
\text { TFBS }\end{array}$ & $A>C$ & $C=4.80$ & $56(90.3)$ & $6(9.70)$ & $0(0.00)$ & $A>C$ & $C=5.60$ & 477 (89.1) & $56(10.5)$ & $2(0.40)$ & 1.000 \\
\hline rs2227532 $2^{\ddagger}$ & IL8 & $\begin{array}{l}\text { Near gene } 5^{\prime} \\
\text { TFBS }\end{array}$ & $T>C$ & $C=9.70$ & $50(80.6)$ & $12(19.4)$ & $0(0.00)$ & $A>G^{+}$ & $G=8.60$ & $448(83.7)$ & $82(15.3)$ & $5(1.00)$ & 0.690 \\
\hline rs $2227538^{\neq}$ & IL8 & $\begin{array}{l}\text { UTR'5 } \\
\text { TFBS, Splicing }\end{array}$ & $C>T$ & $\mathrm{~T}=17.7$ & $41(66.1)$ & $20(32.3)$ & $1(1.60)$ & $G>A^{t}$ & $A=23.2$ & $323(60.4)$ & 176 (32.9) & $36(6.70)$ & 0.291 \\
\hline rs2227545 & IL8 & $\begin{array}{l}\text { UTR'3 } \\
\text { miRNA }\end{array}$ & $A>C$ & $C=8.70$ & 19 (82.6) & $4(17.4)$ & $0(0.00)$ & $A>C$ & $C=8.50$ & 449 (83.9) & $81(15.1)$ & $5(1.00)$ & 0.812 \\
\hline rs2229113 & IL10RA & $\begin{array}{l}\text { Exon } 7 \\
\text { nsSNP, } \\
\text { probably } \\
\text { damaging }\end{array}$ & $G>A$ & $A=20.5$ & 14 (63.7) & $7(31.8)$ & $1(4.50)$ & $G>A$ & $A=18.8$ & $355(66.4)$ & 159 (29.7) & $21(3.90)$ & 0.782 \\
\hline rs2834167 & IL10RB & $\begin{array}{l}\text { Exon } 2 \\
\text { Splicing, } \\
\text { nsSNP, } \\
\text { benign }\end{array}$ & $A>G$ & $G=16.9$ & 44 (71.0) & $15(24.2)$ & $3(4.80)$ & $A>G$ & $G=11.0$ & $423(79.1)$ & 106 (19.8) & $6(1.10)$ & 0.050 \\
\hline rs2856836 & IL1A & $\begin{array}{l}\text { UTR'3 } \\
\text { miRNA }\end{array}$ & $\mathrm{T}>\mathrm{C}$ & $C=17.4$ & 16 (69.6) & $6(26.1)$ & $1(4.30)$ & $A>G^{+}$ & $G=18.6$ & $358(66.9)$ & 155 (29.0) & $22(4.10)$ & 1.000 \\
\hline rs3135932 & IL10RA & $\begin{array}{l}\text { Exon } 5 \\
\text { Splicing, } \\
\text { nsSNP, } \\
\text { benign }\end{array}$ & $A>G$ & $G=2.10$ & $23(95.8)$ & $1(4.20)$ & $0(0.00)$ & $A>G$ & $G=2.60$ & $508(95.0)$ & $26(4.80)$ & $1(0.20)$ & 1.000 \\
\hline rs315951 & IL1RN & $\begin{array}{l}\text { UTR'3 } \\
\text { miRNA }\end{array}$ & $C>G$ & $G=47.9$ & $8(33.3)$ & $9(37.5)$ & $7(29.2)$ & $C>G$ & $G=48.0$ & $144(26.9)$ & $268(50.1)$ & $123(23.0)$ & 0.482 \\
\hline rs3738579 & RNASEL & $\begin{array}{l}\text { UTR'5 } \\
\text { TFBS, Splicing }\end{array}$ & $\mathrm{T}>\mathrm{C}$ & $C=16.7$ & $14(66.7)$ & $7(33.3)$ & $0(0.00)$ & $A>G^{+}$ & $G=12.3$ & 411 (76.8) & $116(21.7)$ & $8(1.50)$ & 0.474 \\
\hline rs3917225 & |L1R1 & $\begin{array}{l}\text { Near gene } 5^{\prime} \\
\text { TFBS }\end{array}$ & $A>G$ & $G=12.3$ & 49 (80.3) & $9(14.8)$ & $3(4.90)$ & $A>G$ & $G=9.10$ & 438 (81.9) & $97(18.1)$ & $0(0.00)$ & 0.001 \\
\hline rs $4073^{\neq}$ & IL8 & $\begin{array}{l}\text { Near gene } 5^{\prime} \\
\text { TFBS }\end{array}$ & $A>T$ & $T=26.6$ & $34(54.8)$ & $23(37.1)$ & $5(8.10)$ & $T>A$ & $A=20.9$ & 335 (62.6) & 176 (32.9) & $24(4.50)$ & 0.262 \\
\hline rs4141134 & IL1R2 & Near gene $5^{\prime}$ & $T>C$ & $C=11.2$ & 39 (79.6) & $9(18.4)$ & $1(2.00)$ & $A>G^{\dagger}$ & $G=13.7$ & 399 (74.6) & $125(23.4)$ & $11(2.00)$ & 0.660 \\
\hline rs4251961 & IL1RN & $\begin{array}{l}\text { Near gene } 5^{\prime} \\
\text { TFBS }\end{array}$ & $T>C$ & $C=20.2$ & 37 (59.7) & $25(40.3)$ & $0(0.00)$ & $A>G^{\dagger}$ & $G=17.9$ & 367 (68.6) & $145(27.1)$ & $23(4.30)$ & 0.035 \\
\hline rs $4252243^{\neq}$ & IL10RA & $\begin{array}{l}\text { Near gene } 5^{\prime} \\
\text { TFBS }\end{array}$ & $C>T$ & $\mathrm{~T}=32.5$ & $9(45.0)$ & $9(45.0)$ & $2(10.0)$ & $G>A^{\dagger}$ & $A=27.5$ & $271(50.7)$ & $234(43.7)$ & $30(5.60)$ & 0.522 \\
\hline
\end{tabular}


Table 1 Functional consequence and prevalence of inflammatory-associated sequence variants (Continued)

\begin{tabular}{|c|c|c|c|c|c|c|c|c|c|c|c|c|c|}
\hline rs4674257 & IL8RB & $\begin{array}{l}\text { Near gene } 5^{\prime} \\
\text { TFBS }\end{array}$ & $G>A$ & $A=25.0$ & $14(58.3)$ & $8(33.3)$ & $2(8.30)$ & $G>A$ & $A=20.1$ & 347 (64.9) & $161(30.10)$ & $27(5.00)$ & 0.468 \\
\hline rs $4674259^{\neq}$ & IL8RB & $\begin{array}{l}\text { UTR'5 } \\
\text { TFBS }\end{array}$ & $A>G$ & $G=23.9$ & $12(52.2)$ & $11(47.8)$ & $0(0.00)$ & $A>G$ & $G=20.0$ & $349(65.0)$ & $158(30.0)$ & $28(5.00)$ & 0.176 \\
\hline rs486907 & RNASEL & $\begin{array}{l}\text { Exon } 1 \\
\text { nsSNP, } \\
\text { benign }\end{array}$ & $G>A$ & $A=16.7$ & $16(66.7)$ & $8(33.3)$ & $0(0.00)$ & $G>A$ & $A=13.2$ & $402(75.1)$ & $125(23.4)$ & $8(1.50)$ & 0.528 \\
\hline rs6726713 & IL1R2 & $\begin{array}{l}\text { Near gene } 5^{\prime} \\
\text { TFBS }\end{array}$ & $C>T$ & $\mathrm{~T}=11.2$ & $38(77.6)$ & $11(22.4)$ & $0(0.00)$ & $G>A^{\dagger}$ & $A=12.1$ & 417 (78.0) & 106 (19.8) & $12(2.20)$ & 0.689 \\
\hline rs $673^{\neq}$ & TNF & $\begin{array}{l}\text { Near gene } 5^{\prime} \\
\text { TFBS }\end{array}$ & $G>A$ & $A=13.7$ & 45 (72.6) & $17(27.4)$ & $0(0.00)$ & $G>A$ & $A=17.4$ & $364(68.0)$ & $156(29.2)$ & $15(2.80)$ & 0.546 \\
\hline rs8178433 & IL10RB & $\begin{array}{l}\text { Near gene } 5^{\prime} \\
\text { TFBS }\end{array}$ & $\mathrm{T}>\mathrm{G}$ & $G=12.9$ & $46(74.2)$ & $16(25.8)$ & $0(0.00)$ & $A>C^{\dagger}$ & $C=12.4$ & $408(76.3)$ & 121 (22.6) & $6(1.10)$ & 0.811 \\
\hline rs949963 & IL1R1 & $\begin{array}{l}\text { Near gene } 5^{\prime} \\
\text { TFBS }\end{array}$ & $G>A$ & $A=31.1$ & $31(50.8)$ & $22(36.1)$ & $8(13.1)$ & $G>A$ & $A=33.1$ & $249(46.5)$ & $218(40.8)$ & 68 (12.7) & 0.771 \\
\hline rs $9610^{\ddagger}$ & IL10RA & $\begin{array}{l}\text { UTR'3 } \\
\text { miRNA }\end{array}$ & $A>G$ & $G=41.9$ & $20(32.3)$ & $32(51.6)$ & $10(16.1)$ & $A>G$ & $G=33.9$ & $237(44.3)$ & $233(43.5)$ & 65 (12.2) & 0.184 \\
\hline rs999788 & IL10RB & $\begin{array}{l}\text { Near gene } 5^{\prime} \\
\text { TFBS }\end{array}$ & $C>T$ & $T=19.5$ & $40(67.8)$ & $15(25.4)$ & $4(6.80)$ & $G>A^{\dagger}$ & $A=12.4$ & 410 (76.6) & 117 (21.9) & $8(1.50)$ & 0.026 \\
\hline
\end{tabular}

${ }^{\dagger}$ The nucleotide change may vary relative to that reported in NCBI depending on whether the genotyping was performed using the sense or anti-sense DNA strand.

${ }^{+T}$ The chi-square test was used to assess differences in the overall genotype frequencies comparing men of African Descent as reported in NCBI to those in the total population from the current study. P-values generated from the Fisher's exact test (in italics) were used when expected genotype counts were $<5$ for either cases or controls.

Abbreviations: MAF Minor Allele Frequency; UTR untranslated region; TFBS transcription factor binding site; nsSNP non-synonymous coding SNP; miRNA microRNA binding site; NCBI National Center for Biotechnology Information Entrez SNP.

${ }^{\ddagger} \mathrm{N} C B \mathrm{~B}$ AFR1 or African American Population Panel.

${ }^{*}$ NCBI ASW Population Panel. 
Table 2 Relationship between inflammatory related sequence variants and prostate cancer risk among men of African Descent

\begin{tabular}{|c|c|c|c|c|c|c|c|c|c|}
\hline Genes & $\begin{array}{l}\text { dbSNP ID location } \\
\text { predicted function }\end{array}$ & Genotype & $\begin{array}{l}\text { Cases } \\
\text { n (\%) }\end{array}$ & $\begin{array}{l}\text { Controls } \\
\text { n (\%) }\end{array}$ & $\begin{array}{l}\text { Unadjusted } \\
\text { OR }(95 \% \mathrm{Cl})^{\dagger}\end{array}$ & $\begin{array}{c}\text { Adjusted } \\
\text { OR }(95 \% \mathrm{Cl})^{\dagger}\end{array}$ & p-value ${ }^{\neq}$ & $p$ trend & $\begin{array}{l}\text { Bonferroni } \\
\text { correction }\end{array}$ \\
\hline \multirow[t]{5}{*}{ IL1R2 } & rs11886877 & GG & 87 (31.2) & $211(39.4)$ & 1.00 (referent) & 1.00 (referent) & 0.034 & 0.010 & NS \\
\hline & Intron 1 & GA & $149(53.4)$ & $265(49.5)$ & $1.36(0.99,1.88)$ & $1.35(0.92,1.98)$ & 0.058 & & \\
\hline & & AA & $43(15.4)$ & $59(11.1)$ & $1.77(1.11,2.82)$ & $1.92(1.11,3.32)$ & 0.017 & & \\
\hline & & $\mathrm{GA}+\mathrm{AA}$ & $192(68.8)$ & $324(60.6)$ & $1.44(1.06,1.95)$ & $1.46(1.01,2.10)$ & 0.021 & & \\
\hline & & $A A$ vs $(G G+G A)$ & & & $1.47(0.96,2.24)$ & $1.61(0.98,2.63)$ & 0.074 & & \\
\hline \multirow[t]{5}{*}{ ILIA } & rs17561 & $\mathrm{CC}$ & 195 (69.9) & $358(66.9)$ & 1.00 (referent) & 1.00 (referent) & 0.025 & 0.108 & NS \\
\hline & Exon 4 & CA & $82(29.4)$ & $155(29.0)$ & $0.97(0.70,1.34)$ & $1.01(0.68,1.48)$ & 0.858 & & \\
\hline & Splicing & AA & $2(0.70)$ & $22(4.10)$ & $0.17(0.04,0.72)$ & $0.40(0.08,1.83)$ & 0.016 & & \\
\hline & nsSNP & $C A+A A$ & $84(30.1)$ & $177(33.1)$ & $0.87(0.64,1.20)$ & $0.96(0.66,1.40)$ & 0.388 & & \\
\hline & benign & $A A$ vs $(C C+C A)$ & & & $0.17(0.04,0.72)$ & $0.40(0.09,1.82)$ & 0.016 & & \\
\hline \multirow[t]{5}{*}{$\operatorname{IL} 8 R B$} & rs11574752 & GG & $230(82.4)$ & $435(81.3)$ & 1.00 (referent) & 1.00 (referent) & 0.011 & 0.784 & NS \\
\hline & 3'-UTR & $\mathrm{GA}$ & $43(15.4)$ & $99(18.5)$ & $0.82(0.55,1.21)$ & $0.90(0.56,1.40)$ & 0.326 & & \\
\hline & miRNA & $\mathrm{AA}$ & $6(2.20)$ & $1(0.20)$ & $11.3(1.36,94.6)$ & $38.4(3.86,382.8)$ & 0.009 & & \\
\hline & & $\mathrm{GA}+\mathrm{AA}$ & 49 (17.6) & $100(18.7)$ & $0.93(0.64,1.35)$ & $1.08(0.69,1.70)$ & 0.693 & & \\
\hline & & $A A$ vs $(G G+G A)$ & & & $11.7(1.40,98.0)$ & $39.2(3.94,390)$ & 0.008 & & \\
\hline \multirow[t]{5}{*}{ TNF } & rs1800629 & GG & $171(61.2)$ & 368 (68.8) & 1.00 (referent) & 1.00 (referent) & 0.047 & 0.087 & NS \\
\hline & 5' near gene & GA & $103(37.0)$ & 153 (28.6) & $1.45(1.06,1.97)$ & $1.54(1.06,2.24)$ & 0.019 & & \\
\hline & TFBS & AA & $5(1.80)$ & $14(2.60)$ & $0.77(0.27,2.16)$ & $1.30(0.37,4.60)$ & 0.619 & & \\
\hline & & $\mathrm{GA}+\mathrm{AA}$ & $108(38.8)$ & $167(31.2)$ & $1.39(1.03,1.90)$ & $1.53(1.06,2.20)$ & 0.032 & & \\
\hline & & $A A$ vs $(G G+G A)$ & & & $0.68(0.24,1.91)$ & $1.13(0.32,3.90)$ & 0.462 & & \\
\hline \multirow[t]{5}{*}{ TNF } & rs673 & GG & $171(61.3)$ & $364(68.0)$ & 1.00 (referent) & 1.00 (referent) & 0.009 & 0.228 & NS \\
\hline & 5' near gene & GA & $106(38.0)$ & $156(29.2)$ & $1.45(1.06,2.00)$ & $1.50(1.04,2.16)$ & 0.018 & & \\
\hline & TFBS & $\mathrm{AA}$ & $2(0.70)$ & $15(2.80)$ & $0.28(0.06,1.26)$ & $0.47(0.09,2.40)$ & 0.097 & & \\
\hline & & $\mathrm{GA}+\mathrm{AA}$ & 108 (39.1) & $171(32.0)$ & $1.34(0.99,1.82)$ & $1.43(1.00,2.05)$ & 0.055 & & \\
\hline & & $A A$ vs $(G G+A G)$ & & & $0.25(0.06,1.10)$ & $0.41(0.08,2.07)$ & 0.067 & & \\
\hline \multirow[t]{5}{*}{ ILIA } & rs2856836 & AA & $196(70.3)$ & $358(66.9)$ & 1.00 (referent) & 1.00 (referent) & 0.024 & 0.089 & NS \\
\hline & 3'-UTR & $A G$ & $81(29.0)$ & $155(29.0)$ & $0.96(0.69,1.32)$ & $0.99(0.67,1.45)$ & 0.776 & & \\
\hline & miRNA & GG & $2(0.70)$ & $22(4.10)$ & $0.17(0.04,0.71)$ & $0.40(0.09,1.82)$ & 0.016 & & \\
\hline & & $A G+G G$ & $83(29.7)$ & 177 (33.1) & $0.86(0.63,1.17)$ & $0.94(0.65,1.36)$ & 0.333 & & \\
\hline & & $\mathrm{GG}$ vs $(A A+A G)$ & & & $0.17(0.04,0.72)$ & $0.40(0.09,1.82)$ & 0.016 & & \\
\hline \multirow[t]{5}{*}{ ILIORA } & rs4252243 & GG & $134(48.4)$ & $268(50.4)$ & 1.00 (referent) & 1.00 (referent) & 0.066 & 0.168 & NS \\
\hline & 5' near gene & GA & $115(41.5)$ & $234(44.0)$ & $0.98(0.72,1.32)$ & $0.83(0.58,1.18)$ & 0.893 & & \\
\hline & TFBS & AA & $28(10.1)$ & $30(5.60)$ & $1.86(1.07,3.24)$ & $1.62(0.82,3.21)$ & 0.028 & & \\
\hline & & $\mathrm{GA}+\mathrm{AA}$ & 143 (51.6) & 264 (49.6) & $1.08(0.81,1.44)$ & $0.91(0.64,1.28)$ & 0.605 & & \\
\hline & & $A A$ vs $(G G+G A)$ & & & $1.88(1.10,3.21)$ & $1.77(0.91,3.43)$ & 0.021 & & \\
\hline
\end{tabular}

${ }^{\ddagger}$ On a separate line before the text regarding the chi-square test $\mathrm{p}$-values state the following:

${ }^{\dagger}$ Boldface odd ratios (ORs) and $95 \%$ confidence interval $(\mathrm{Cl})$ indicate a significant relationship between the selected SNPs and prostate cancer risk. From top to bottom within the column, the chi-square test $\mathrm{p}$-values were used to determine the difference in the genotype frequencies between cases and controls for the overall, minor/major versus major/major genotypes, as well as the dominant (i.e., minor/minor versus major/major), co-dominant (minor/minor + major/minor versus major/major), and recessive genetic models (minor/minor versus major/major + major/minor). P-values generated from the Fisher's Exact test (in italics) were calculated when expected genotype counts were $<5$ for either cases or controls. Statistically significant $\mathrm{p}$-values are marked in bold face. Abbreviations: UTR, untranslated region; TFBS, transcription factor binding site; miRNA, microRNA binding site; NS, non-significant. 
Table 3 Relationship between inflammatory related sequence variants and prostate cancer risk among U.S. and Jamaican men

\begin{tabular}{|c|c|c|c|c|c|c|c|c|c|c|}
\hline Genes & $\begin{array}{l}\text { dbSNP ID location } \\
\text { predicted function }\end{array}$ & Genotype & $\begin{array}{l}\text { Unadjusted OR } \\
(95 \% \mathrm{Cl}) \text { US ment }\end{array}$ & $\begin{array}{l}\text { Age-adjusted OR } \\
(95 \% \mathrm{Cl}) \text { US ment }\end{array}$ & $\begin{array}{c}\text { Unadjusted OR } \\
(95 \% \mathrm{Cl}) \text { Jamaican ment }\end{array}$ & $\begin{array}{c}\text { Age-adjusted OR } \\
(95 \% \mathrm{Cl}) \text { Jamaican ment }\end{array}$ & $\begin{array}{l}\text { p-value } \\
\text { US men }\end{array}$ & $\begin{array}{c}\text { p-value } \\
\text { Jamaican men }\end{array}$ & $\begin{array}{l}\text { p-trend } \\
\text { US men }\end{array}$ & $\begin{array}{c}\text { p-trend } \\
\text { Jamaican men }\end{array}$ \\
\hline \multirow[t]{5}{*}{ ILIB } & rs1071676 & GG & 1.00 (referent) & 1.00 (referent) & 1.00 (referent) & 1.00 (referent) & 0.050 & 0.550 & 0.022 & 0.276 \\
\hline & UTR'3 & GC & $0.72(0.48,1.10)$ & $0.70(0.42,1.14)$ & $1.39(0.71,2.70)$ & $1.28(0.62,2.64)$ & 0.124 & 0.338 & & \\
\hline & miRNA & CC & $0.16(0.02,1.25)$ & $0.19(0.02,2.00)$ & $2.02(0.18,22.8)$ & $1.15(0.10,14.6)$ & 0.035 & 0.500 & & \\
\hline & & $\mathrm{GC}+\mathrm{CC}$ & $0.66(0.44,1.00)$ & $0.66(0.40,1.10)$ & $1.42(0.74,2.72)$ & $1.26(0.62,2.60)$ & 0.050 & 0.294 & & \\
\hline & & $C C$ vs $(G G+G C)$ & $0.18(0.02,1.36)$ & $0.21(0.02,2.18)$ & $1.89(0.16,21.1)$ & $1.10(0.08,13.7)$ & 0.046 & 0.525 & & \\
\hline \multirow[t]{5}{*}{ ILIB } & rs1143634 & GG & 1.00 (referent) & 1.00 (referent) & 1.00 (referent) & 1.00 (referent) & 0.051 & 0.447 & 0.016 & 0.203 \\
\hline & Exon 4 & GA & $0.67(0.44,1.01)$ & $0.65(0.40,1.06)$ & $1.51(0.76,3.00)$ & $1.37(0.64,2.90)$ & 0.058 & 0.243 & & \\
\hline & Splicing & AA & $0.21(0.02,1.60)$ & $0.24(0.02,2.86)$ & $2.05(0.18,23.0)$ & $1.16(0.10,14.6)$ & 0.080 & 0.496 & & \\
\hline & & $\mathrm{GA}+\mathrm{AA}$ & $0.63(0.42,0.95)$ & $0.62(0.38,1.02)$ & $1.54(0.78,3.00)$ & $1.36(0.65,2.82)$ & 0.028 & 0.208 & & \\
\hline & cds-synonymous & $A A$ vs $(G G+G A)$ & $0.23(0.02,1.80)$ & $0.27(0.02,3.20)$ & $1.89(0.16,21.1)$ & $1.10(0.08,13.7)$ & 0.105 & 0.525 & & \\
\hline \multirow[t]{5}{*}{ ILIR2 } & rs11886877 & GG & 1.00 (referent) & 1.00 (referent) & 1.00 (referent) & 1.00 (referent) & 0.007 & 0.889 & 0.002 & 0.631 \\
\hline & Intron 1 & GA & $1.60(1.08,2.40)$ & $1.63(1.00,2.64)$ & $0.92(0.50,1.68)$ & $0.94(0.48,1.80)$ & 0.020 & 0.782 & & \\
\hline & & AA & $2.34(1.31,4.16)$ & $2.75(1.38,5.50)$ & $0.82(0.36,1.86)$ & $0.94(0.38,2.30)$ & 0.004 & 0.633 & & \\
\hline & & $\mathrm{GA}+\mathrm{AA}$ & $1.72(1.18,2.52)$ & $1.82(1.14,2.88)$ & $0.89(0.50,1.58)$ & $0.94(0.50,1.74)$ & 0.005 & 0.700 & & \\
\hline & & $A A$ vs $(G G+G A)$ & $1.76(1.04,2.96)$ & $2.05(1.10,3.80)$ & $0.86(0.40,1.80)$ & $0.97(0.43,2.20)$ & 0.033 & 0.691 & & \\
\hline \multirow[t]{5}{*}{ RNASEL } & rs12135247 & AA & 1.00 (referent) & 1.00 (referent) & 1.00 (referent) & 1.00 (referent) & 0.800 & 0.025 & 0.909 & 0.216 \\
\hline & UTR'3 & $A G$ & $1.06(0.72,1.58)$ & $1.14(0.71,1.84)$ & $2.17(1.14,4.12)$ & $2.10(1.04,4.24)$ & 0.756 & 0.018 & & \\
\hline & TFBS & GG & $0.77(0.30,1.96)$ & $0.70(0.24,2.10)$ & $0.45(0.08,2.40)$ & $0.28(0.04,1.70)$ & 0.570 & 0.284 & & \\
\hline & miRNA & $A G+G G$ & $1.02(0.70,1.50)$ & $1.07(0.68,1.68)$ & $1.81(0.99,3.30)$ & $1.68(0.88,3.24)$ & 0.906 & 0.053 & & \\
\hline & & $G G$ vs $(A G+A A)$ & $0.76(0.30,1.92)$ & $0.67(0.22,1.97)$ & $0.36(0.06,1.91)$ & $0.22(0.04,1.35)$ & 0.555 & 0.196 & & \\
\hline \multirow[t]{5}{*}{ TNF } & rs1800629 & GG & 1.00 (referent) & 1.00 (referent) & 1.00 (referent) & 1.00 (referent) & 0.101 & 0.782 & 0.113 & 0.549 \\
\hline & $5^{\prime}$ near gene & GA & $1.50(1.03,2.20)$ & $1.52(0.96,2.42)$ & $1.21(0.68,2.12)$ & $1.41(0.78,2.63)$ & 0.034 & 0.518 & & \\
\hline & TFBS & AA & $0.90(0.28,2.80)$ & $1.51(0.36,6.24)$ & $1.00(0.06,16.3)$ & $1.00(0.06,17.2)$ & 0.551 & 0.752 & & \\
\hline & & $\mathrm{GA}+\mathrm{AA}$ & $1.44(0.99,2.10)$ & $1.53(0.97,2.40)$ & $1.20(0.68,2.10)$ & $1.40(0.75,2.60)$ & 0.050 & 0.525 & & \\
\hline & & $A A$ vs $(G G+G A)$ & $0.78(0.25,2.42)$ & $1.32(0.32,5.40)$ & $0.94(0.06,15.2)$ & $0.88(0.05,15.0)$ & 0.452 & 0.734 & & \\
\hline \multirow[t]{5}{*}{ ILTA } & rs1800587 & GG & 1.00 (referent) & 1.00 (referent) & 1.00 (referent) & 1.00 (referent) & 0.088 & 0.450 & 0.028 & 0.224 \\
\hline & UTR'5 & GA & $0.75(0.50,1.10)$ & $0.68(0.42,1.08)$ & $1.38(0.76,2.50)$ & $1.42(0.74,2.72)$ & 0.144 & 0.297 & & \\
\hline & TFBS & AA & $0.56(0.32,0.96)$ & $0.78(0.40,1.53)$ & $1.56(0.69,3.50)$ & $1.64(0.70,4.00)$ & 0.038 & 0.279 & & \\
\hline & Splicing (ESE or & $\mathrm{GA}+\mathrm{AA}$ & $0.70(0.48,1.00)$ & $0.70(0.45,1.10)$ & $1.42(0.80,2.50)$ & $1.47(0.80,2.72)$ & 0.053 & 0.222 & & \\
\hline & ESS) & $A A$ vs $(G G+G A)$ & $0.66(0.40,1.10)$ & $0.96(0.52,1.80)$ & $1.30(0.62,2.70)$ & $1.34(0.60,3.00)$ & 0.105 & 0.478 & & \\
\hline ILIORA & rs4252243 & GG & 1.00 (referent) & 1.00 (referent) & 1.00 (referent) & 1.00 (referent) & 0.062 & 0.620 & 0.275 & 0.329 \\
\hline
\end{tabular}


Table 3 Relationship between inflammatory related sequence variants and prostate cancer risk among U.S. and Jamaican men (Continued)

\begin{tabular}{|c|c|c|c|c|c|c|c|c|c|c|}
\hline & 5' near gene & GA & $0.92(0.63,1.33)$ & $0.70(0.44,1.10)$ & $1.24(0.70,2.20)$ & $1.21(0.64,2.28)$ & 0.648 & 0.448 & & \\
\hline & TFBS & AA & $2.02(1.04,3.95)$ & $2.10(0.90,4.98)$ & $1.50(0.54,4.26)$ & $1.04(0.34,3.20)$ & 0.038 & 0.436 & & \\
\hline & & $\mathrm{GA}+\mathrm{AA}$ & $1.03(0.72,1.50)$ & $0.81(0.52,1.30)$ & $1.28(0.74,2.21)$ & $1.15(0.64,2.10)$ & 0.863 & 0.328 & & \\
\hline & & $A A$ vs $(G G+G A)$ & $2.11(1.10,4.02)$ & $2.49(1.08,5.72)$ & $1.37(0.50,3.74)$ & $1.02(0.40,2.98)$ & 0.023 & 0.539 & & \\
\hline \multirow[t]{5}{*}{ TNF } & rs673 & GG & 1.00 (referent) & 1.00 (referent) & 1.00 (referent) & 1.00 (referent) & 0.027 & 0.452 & 0.279 & 0.874 \\
\hline & 5' near gene & GA & $1.50(1.02,2.20)$ & $1.46(0.92,2.30)$ & $1.22(0.70,2.14)$ & $1.41(0.76,2.62)$ & 0.025 & 0.498 & & \\
\hline & TFBS & AA & $0.24(0.03,1.84)$ & $0.54(0.06,4.44)$ & $0.33(0.03,3.24)$ & $0.40(0.03,4.70)$ & 0.116 & 0.315 & & \\
\hline & & $\mathrm{GA}+\mathrm{AA}$ & $1.38(0.95,2.00)$ & $1.40(0.88,2.20)$ & $1.14(0.66,2.00)$ & $1.33(0.72,2.46)$ & 0.087 & 0.635 & & \\
\hline & & $A A$ vs $(G G+G A)$ & $0.21(0.02,1.60)$ & $0.47(0.06,3.91)$ & $0.31(0.03,2.98)$ & $0.35(0.03,4.08)$ & 0.080 & 0.286 & & \\
\hline
\end{tabular}

On a separate line before the text regarding the chi-square test $\mathrm{p}$-values state the following:

+Boldface odd ratios (ORs) and $95 \%$ confidence interval (CI) indicate a significant relationship between the selected SNPs and prostate cancer risk.

${ }^{*}$ From top to bottom within the column, the chi-square test $\mathrm{p}$-values were used to determine the difference in the genotype frequencies between cases and controls for the overall, minor/major versus major/major genotypes, as well as the dominant (i.e., minor/minor versus major/major), co-dominant (minor/minor + major $/ \mathrm{minor}$ versus major/major), and recessive genetic models (minor/minor versus major/major + major/minor). P-values generated from the Fisher's Exact test (in italics) were calculated when expected genotype counts were $<5$ for either cases or controls. Statistically significant p-values are marked in bold face. Abbreviations: UTR, untranslated region; TFBS, transcription factor binding site; cds-syn, synonymous SNP; miRNA, microRNA binding site. 
mesenchymal transition (EMT), by binding to IL1R2 to increase prostate cancer tumorigenesis [30].

Out of 44 inflammatory-related sequence variants, 7 SNPs included in our study were evaluated in relation to prostate cancer outcomes within 4 independent observational studies $[6,7,10,11]$. Commensurate with our findings, two observational studies demonstrated that sequence variants detected in IL10 (rs1800871, rs1800872) and IL8 rs4073 were not significantly related to prostate cancer risk $[6,11]$. Inheritance of the TNF rs1800629 AA genotype was associated with a significant 1.8 fold increase in prostate cancer risk among Caucasian men in a small study (150 cases, 150 controls); however, this marker resulted in null findings in a larger study (468 cases, 468 controls) [6,7]. In our preliminary analyses, inheritance of one or more TNF rs1800629 A alleles was marginally associated with a 1.5-fold increase in prostate risk; however, this relationship did not survive adjustment after multiple hypothesis testing. Lastly, IL1O rs1800896 G and IL1B rs1143627 C alleles had protective effects in two separate Caucasian subpopulations. However, neither of these markers were significantly related to prostate cancer among African-American men in the current study. Casey and colleagues (2002) showed a 2-fold increase in prostate cancer susceptibility linked to inheritance of the RNASEL rs486907 AA genotype among mostly men of European descent [10]. This locus was not related to prostate cancer risk among African-Americans in the current study. Racial/ethnic disparities in the aforementioned risk estimates may be attributed to differences in minor allele frequencies, failure to adjust findings for multiple hypothesis testing or inadequate sample size among men with African ancestry.

In this study, we considered the strengths, limitations and future directions of the project. Forty-four sequence variants were evaluated in relation to prostate cancer risk among men of African Descent from the U.S. and Jamaica. Upon stratification by study center, the IL1R2 rs11886877 locus was marginally related to prostate cancer among men of African descent from the U.S. However, overall the inflammatory-related sequence variants were not robustly related to prostate cancer among our study participants. Despite this, we cannot eliminate the possibility that IL1R2 and other inflammatory-related sequence variants not included in this study may influence the risk of prostate cancer development or aggressive tumor behavior. In larger studies, the impact of individual or interaction among inflammatory cytokine-associated sequence variants in relation to prostate cancer tumor grade, biochemical or disease recurrence, and mortality using targeted sequencing, in vitro studies, in silico and bioinformatics tools. Such efforts will help to identify genetic markers linked to disproportionately high prostate cancer incidence, mortality, and morbidity rates among men of African Descent. Population admixture, which commonly occurs among men of African descent, may bias risk estimates. However, adjustment of risk estimates by West African Ancestry and/or age did not significantly modify the directionality of observed risk estimates among men from the U.S. (data not shown). Although, the sample size of this study population is small, there was ample statistical power to accurately detect risk estimates, ranging between $1.4-1.8$ or $0.55-0.70$. Our findings are important to genetic epidemiology research teams interested in pooling genetic and tumor characteristic data to determine whether other variant inflammatory-related cytokines contribute to prostate cancer susceptibility and disease prognosis. Although this study displays a modest association between inflammatory-related cytokine variant IL1R2 rs11886877 and prostate cancer risk, this relationship has yet to be tested biologically. The association of IL1R2 rs11886877 with prostate cancer risk may prove to be strong in a larger study population.

\section{Conclusions}

Chronic inflammation is an established risk factor of prostate cancer and many studies argue that it can lead to prostate cancer development. In this study, 44 inflammatory-related cytokine variants that may play a role in chronic inflammation were analyzed in relation to prostate cancer risk. Our preliminary data suggests that the possession of IL1R2 rs11886877 locus modifies prostate cancer susceptibility among individuals with African ancestry in the U.S. However, the association of the IL1R2 variant with prostate risk did not remain significant after adjust for multiple hypothesis testing. Future studies with ample statistical power to accommodate adjustment for multiple comparisons bias, will enable us to evaluate the impact of the IL1R2 variant or a combination of inflammatory cytokine SNPs in relation to prostate cancer risk, tumor grade, biochemical or disease recurrence, and mortality. These studies will lead to the identification of genetic markers that modify the susceptibility of individuals.

\section{Abbreviations}

SNP: Single nucleotide polymorphism; LR: Logistic regression.

\section{Competing interests}

The authors declare that they have no competing interests.

\section{Authors' contributions}

LRK and KSK: conceptualized the project. LRK, KSK, CR, MJ, NM, MT, SM: participated in the study design. DZJ, LRK: composed the manuscript. DZJ, LRK, CR, REF: revised subsequent manuscript drafts. DZJ, NCK: quality contro and statistical analysis. LRK: supervised quality control analysis, data-management and statistical analysis. LRK, DZJ, CR, KSK: interpreted the data, gave important intellectual input toward the introduction, results and/or discussion. All co-authors: read and edited the manuscript drafts as well as gave final approval of the final manuscript draft.

\section{Acknowledgements}

We thank Tiva T. VanCleave and Nicole A. Lavender for DNA sample preparation. We appreciate the contract services of Expression Analysis, Inc. (http://www.expressionanalysis.com) for the generation of genotype data. 
We offer gratitude to Dr. Rick Kittles for the donation of DNA samples from prostate cancer patients.

Lastly, we value Peter Andrews for his service as a computer programming consultant on this project.

Grant/Research support: Clinical Translational Science Pilot Grant to LRK; the JGBCC Bucks for Brains "Our Highest Potential" in Cancer Research Endowment to LRK; P20-MD000175 NIH NCMHD to KSK.

\section{Author details}

'Department of Pharmacology \& Toxicology, University of Louisville, Louisville, KY, USA. ${ }^{2}$ Cancer Prevention and Control Program, Fox Chase Cancer Center, Philadelphia, PA, USA. ${ }^{3}$ Molecular and Cellular Biology Program, State University of New York, Brooklyn, NY, USA. ${ }^{4}$ Department of Community Health and Psychiatry, University of West Indies, Mona, Kingston, Jamaica. ${ }^{5}$ Department of Basic Medical Sciences, University of the West Indies, Mona Campus, Kingston, Jamaica. ${ }^{6}$ Tropical Medicine Research Institute, University of the West Indies, Mona, Kingston, Jamaica. 'Biomedical/ Biotechnology Research Institute (BBRI), North Carolina Central University, Durham, NC, USA.

\section{Received: 29 July 2013 Accepted: 3 December 2013}

Published: 23 December 2013

\section{References}

1. Coussens LM, Werb Z: Inflammation and cancer. Nature 2002, 420(6917):860-867.

2. Vasto S, Carruba G, Candore G, Italiano E, Di Bona D, Caruso C: Inflammation and prostate cancer. Future Oncol 2008, 4(5):637-645

3. Sfanos KS, De Marzo AM: Prostate cancer and inflammation: the evidence. Histopathology 2012, 60(1):199-215.

4. Veitonmaki T, Tammela TL, Auvinen A, Murtola TJ: Use of aspirin, but not other non-steroidal anti-inflammatory drugs is associated with decreased prostate cancer risk at the population level. Eur J Cancer 2013, 49(4):938-945.

5. Feghali CA, Wright TM: Cytokines in acute and chronic inflammation. Front Biosci 1997, 2:d12-d26.

6. Dluzniewski PJ, Wang MH, Zheng SL, De Marzo AM, Drake CG, Fedor HL, Partin AW, Han M, Fallin MD, XU J, et al: Variation in IL10 and other genes involved in the immune response and in oxidation and prostate cancer recurrence. Cancer Epidemiol Biomarkers Prev 2012, 21(10):1774-1782.

7. Berhane N, Sobti RC, Melesse S, Mahdi SA, Kassu A: Significance of Tumor necrosis factor alpha-308 (G/A) gene polymorphism in the development of prostate cancer. Mol Biol Rep 2012, 39(12):11125-11130.

8. Lin DW, FitzGerald LM, Fu R, Kwon EM, Zheng SL, Kolb S, Wiklund F, Stattin $P$, Isaacs WB, Xu J, et al: Genetic variants in the LEPR, CRY1, RNASEL, IL4, and ARVCF genes are prognostic markers of prostate cancer-specific mortality. Cancer Epidemiol Biomarkers Prev 2011, 20(9):1928-1936.

9. Alvarez-Cubero MJ, Saiz M, Martinez-Gonzalez LJ, Alvarez JC, Lorente JA, Cozar JM: RNASEL study of genetics of prostate cancer and its relation to clinical staging. Actas Urol Esp 2012, 36(5):306-311.

10. Casey G, Neville PJ, Plummer SJ, Xiang Y, Krumroy LM, Klein EA, Catalona WJ, Nupponen N, Carpten JD, Trent JM, et al: RNASEL Arg462Gln variant is implicated in up to $13 \%$ of prostate cancer cases. Nat Genet 2002, 32(4):581-583.

11. Kesarwani P, Ahirwar DK, Mandhani A, Singh AN, Dalela D, Srivastava AN, Mittal RD: IL-10-1082 G > A: a risk for prostate cancer but may be protective against progression of prostate cancer in North Indian cohort. World J Urol 2009, 27(3):389-396.

12. Tindall EA, Severi G, Hoang HN, Southey MC, English DR, Hopper JL, Giles GG, Hayes VM: Interleukin-6 promoter variants, prostate cancer risk, and survival. Prostate 2012, 72(16):1701-1707.

13. Tindall EA, Severi G, Hoang HN, Ma CS, Fernandez P, Southey MC, English DR, Hopper JL, Heyns CF, Tangye SG, et al: Comprehensive analysis of the cytokine-rich chromosome 5q31.1 region suggests a role for IL-4 gene variants in prostate cancer risk. Carcinogenesis 2010, 31(10):1748-1754.

14. American Cancer S: Cancer Facts and Figures 2012. American Cancer Society: Atlanta, Georgia; 2012.

15. American Cancer S: Cancer Facts \& Figures for African Americans 2011-2012. American Cancer Society: Atlanta; 2011.
16. Zabaleta J, Lin HY, Sierra RA, Hall MC, Clark PE, Sartor OA, Hu JJ, Ochoa AC: Interactions of cytokine gene polymorphisms in prostate cancer risk. Carcinogenesis 2008, 29(3):573-578.

17. Zabaleta J, Su LJ, Lin HY, Sierra RA, Hall MC, Sartor AO, Clark PE, Hu JJ, Ochoa AC: Cytokine genetic polymorphisms and prostate cancer aggressiveness. Carcinogenesis 2009, 30(8):1358-1362.

18. Kidd LR, Jones DZ, Rogers EN, Kidd NC, Beache S, Rudd JE, Ragin C, Jackson M, McFarlane-Anderson N, Tulloch-Reid M, et al: Chemokine Ligand 5 (CCL5) and chemokine receptor (CCR5) genetic variants and prostate cancer risk among men of African Descent: a case-control study. Hereditary Cancer Clin Prac 2012, 10(1):16.

19. Jackson MD, Walker SP, Simpson-Smith CM, Lindsay CM, Smith G, McFarlane-Anderson N, Bennett Fl, Coard KC, Aiken WD, Tulloch T, et al: Associations of whole-blood fatty acids and dietary intakes with prostate cancer in Jamaica. Cancer Causes Control 2012, 23(1):23-33.

20. Manolio TA, Collins FS, Cox NJ, Goldstein DB, Hindorff LA, Hunter DJ, McCarthy MI, Ramos EM, Cardon LR, Chakravarti A, et al: Finding the missing heritability of complex diseases. Nature 2009, 461(7265):747-753.

21. McCarthy MI, Abecasis GR, Cardon LR, Goldstein DB, Little J, loannidis JP, Hirschhorn JN: Genome-wide association studies for complex traits: consensus, uncertainty and challenges. Nat Rev Genet 2008, 9(5):356-369.

22. Steemers FJ, Gunderson KL: Whole genome genotyping technologies on the Bead Array platform. Biotechnol J 2007, 2(1):41-49.

23. Menashe I, Rosenberg PS, Chen BE: PGA: power calculator for case-control genetic association analyses. BMC Genet 2008, 9:36.

24. Song N, Han S, Lee KM, Choi JY, Park SK, Jeon S, Lee Y, Ahn HS, Shin HY, Kang $\mathrm{HJ}$, et al: Genetic variants in interleukin-2 and risk of lymphoma among children in Korea. Asian Pac J Cancer Prev 2012, 13(2):621-623.

25. Gonzalez CA, Sala N, Capella G: Genetic susceptibility and gastric cancer risk. Int J Cancer 2002, 100(3):249-260.

26. Lin HC, Liu CC, Kang WY, Yu CC, Wu TT, Wang JS, Wu WJ, Huang CH, Wu MT, Huang SP: Influence of cytokine gene polymorphisms on prostate-specific antigen recurrence in prostate cancer after radical prostatectomy. Urol Int 2009, 83(4):463-470.

27. Leshem O, Madar S, Kogan-Sakin I, Kamer I, Goldstein I, Brosh R, Cohen Y, Jacob-Hirsch J, Ehrlich M, Ben-Sasson S, et al: TMPRSS2/ERG promotes epithelial to mesenchymal transition through the ZEB1/ZEB2 axis in a prostate cancer model. PLoS One 2011, 6(7):e21650.

28. Chang SY, Su PF, Lee TC: Ectopic expression of interleukin-1 receptor type II enhances cell migration through activation of the pre-interleukin 1alpha pathway. Cytokine 2009, 45(1):32-38.

29. Ricote M, Garcia-Tunon I, Bethencourt FR, Fraile B, Paniagua R, Royuela M: Interleukin-1 (IL-1alpha and IL-1beta) and its receptors (IL-1RI, IL-1RII, and IL-1Ra) in prostate carcinoma. Cancer 2004, 100(7):1388-1396.

30. King JC, Xu J, Wongvipat J, Hieronymus H, Carver BS, Leung DH, Taylor BS, Sander C, Cardiff RD, Couto SS, et al: Cooperativity of TMPRSS2-ERG with PI3-kinase pathway activation in prostate oncogenesis. Nat Genet 2009, 41(5):524-526.

\section{doi:10.1186/1897-4287-11-19}

Cite this article as: Jones et al:: The impact of genetic variants in inflammatory-related genes on prostate cancer risk among men of African Descent: a case control study. Hereditary Cancer in Clinical Practice 2013 11:19.

\section{Submit your next manuscript to BioMed Central and take full advantage of:}

- Convenient online submission

- Thorough peer review

- No space constraints or color figure charges

- Immediate publication on acceptance

- Inclusion in PubMed, CAS, Scopus and Google Scholar

- Research which is freely available for redistribution 